\title{
Paper Digital Predistortion of Wideband Signals with Reduced Complexity Based on Feedback Wiener System
}

\author{
Tayeb H. C. Bouazza, Smail Bachir, and Claude Duvanaud \\ XLIM Laboratory UMR-CNRS 7252, Institute of Technology of Angoulême, University of Poitiers, Angoulême, France
}

https://doi.org/10.26636/jtit.2021.144520

\begin{abstract}
Digital predistortion (DPD) using baseband signals is commonly used for power amplifier linearization. This paper is devoted to this subject and aims to reduce DPD complexity. In this study, we propose a structure that allows to decrease the number of DPD parameters by using multiple blocks, with each one of them dedicated to characterizing the non-linear behavior and/or memory effects. Such a structure is based on the feedback Wiener system, involving a FIR filter used as a feedback path to reproduce the PA inverse dynamics. A memory polynomial block (MP) is inserted as the final element to minimize the modeling errors. A relevant model identification method, based on an iterative algorithm, has been developed as well. The proposed architecture is used for the linearization of a commercial class-AB LDMOS RF PA by NXP Semiconductors, in wideband communication systems. Comparison of performance with the conventional generalized memory polynomial model (GMP) shows that the proposed model offers similar results, with its advantage consisting in the reduced number of parameters.
\end{abstract}

Keywords-digital predistortion, feedback Wiener model, GMP mode, parameter identification, power amplifier.

\section{Introduction}

The key challenge in the design of radio frequency (RF) power amplifiers (PA) is to achieve high efficiency characteristics by using transistors at their near-to-saturation point [1]. Under such operating conditions, PA nonlinearities and memory effects create significant signal distortions in both time and frequency domains, such as, for instance, scattered constellations and asymmetries in spectral regrowth [2]-[5]. These effects are more pronounced in the case of high-power fluctuations in multi-band and multi-carrier signals [6], [7]. So, such a behavior degrades the transmitter's efficiency and decreases transmission quality. One solution relied upon to minimize these effects, while simultaneously respecting spectral masks and without compromising efficiency, is to apply linearization techniques.
Several linearization techniques have been developed to mitigate PA non-linearities at high levels, and consequently to improve PA linearity versus power efficiency trade-off. Predistortion methods have been proposed as a solution with high potential to overcome nonlinear effects [8]-[10]. These techniques aim to introduce inverse non-linearities that compensate the PA gain, as well as phase and memory effects distortions [11], [12]. Depending on the position of the predistorter and on the provided signals, three types of predistortion techniques may be distinguished: those applied in RF [11], in intermediate frequencies (IF) [13] and in baseband (BB) [14]. From all linearization techniques referred to above, baseband digital predistortion (DPD) receives the most attention. It is widely deployed in modern wireless systems, as it allows to achieve good linearization performance through the use of reduced sampling frequency, without additional RF elements, and is, therefore, more cost effective.

In DPD and due to the complexity of the PA behavior, nonlinear mathematical functions are required to sufficiently describe the inverse of PA characteristics [15]. In the stateof-the-art, the commonly used models are derived from the Volterra series [16]-[18]. Among them, one may distinguish the memory polynomial (MP) model [19], [20], the generalized memory polynomial (GMP) model [21] or the non-linear auto-regressive moving average (NARMA) model [22]. Other models, such as the block-oriented nonlinear system (for instance Hammerstein and Wiener) [23], vector-switched models [24], decomposed vector rotation models [25], and neural network models [14] are used as well.

The use of a large number of terms is suitable for making the DPD more accurate, but unfortunately, this comes at the cost of a complicated implementation and long lead times required to estimate the coefficients. In this study, we focus on reducing the number of the model's parameters and we propose to study and use the feedback Wiener (FW) model [23] as a predistorter. To generate PA memory effects, a filter block with time delays is used in its 
feedback path. A low order MP model may also be cascaded with the FW block for modeling errors in wideband applications [26]. The discussed DPD function using the proposed structure, referred to, in this paper, the feedback Wiener with memory polynomial (FWMP), and its identification algorithm are presented and tested using a 2-stage $20 \mathrm{~W}$ class-AB LDMOS RF PA by NXP Semiconductors. Studies concerned with model complexity and focusing on optimizing the number of model coefficients and drawing comparisons with the performance of the GMP model show a good compromise between linearization accuracy and model complexity.

This paper is organized as follows. In Section 2, we introduce a new, less complex, block-oriented model based on the feedback Wiener system. The identification process of a predistorter using the proposed FWMP model is described in Section 3. Linearization performance experimental tests using the proposed structure and a comparison with the MP and GMP models are described in Section 4. Finally, conclusions and some perspectives are given in Section 5 .

\section{Block-oriented Model Description}

A block-oriented model will be used in this study for the implementation of the predistorter (Fig. 1). It has been established by relying on the circuit-based approach, allowing to take into account the fundamental non-linear properties, memory effects and the bilateral behavior of the active devices [26].

As shown in Fig. 1, this structure is based on a combination of two blocks: a feedback Wiener system which models the main PA behavior, i.e. interaction between non-linearities and memory effects, and an MP model for the remaining modeling errors. The FW block itself is made up of two sub-blocks: a feed-forward memory-less non-linearity and a feedback finite impulse response (FIR) filter, where $q^{-1}$ is the unit time delay.
The main signals of the FWMP model may be formulated as:

$x(n)$ is the output of the FW system, such as:

$$
x(n)=\sum_{p=1}^{P} c_{p} \cdot w(n)^{p}=\sum_{p=1}^{P} c_{p} \cdot\left[g_{0} \cdot M_{i n}(n)-d(n)\right]^{p}
$$

where $c_{p}$ are the non-linear terms of the non-linearity function, $g_{0}$ is the complex gain and $P$ is the non-linearity order. $M_{\text {in }}$ is the model input. $F(\omega)$ is a FIR filter and its output $d(n)$ may be formulated as:

$$
d(n)=\sum_{m=1}^{M} b_{m} \cdot x(n-m),
$$

where $M$ is the memory depth of the FIR filter. Signal $x(n)$ is used as an input for the MP model:

$$
M_{\text {out }}(n)=\sum_{p=0}^{P_{a}-1} \sum_{m=0}^{M_{a}-1} a_{p m} \cdot x(n-m) \cdot|x(n-m)|^{p},
$$

where $M_{\text {out }}$ is the model output, while $P_{a}$ and $M_{a}$ are the non-linearity order and memory depth, respectively.

Note that, since non-linearities and memory effects are treated separately in the FW block, the proposed model has the advantage of an additive evolution in its first block, meaning that after incrementing a parameter in the FW block, only a single increment in the number of model coefficients occurs. As a result, the total number of coefficients is given by:

$$
N_{F W M P}=\overbrace{(P+M)}^{F W}+\overbrace{\left(P_{a} \times M_{a}\right)}^{M P} .
$$

\section{DPD using FWMP Model}

The DPD is based on the estimation of the inverse PA characteristics to compensate its static and dynamic nonlinearities. In the case of the proposed model, Fig. 2 shows the principle of the off-line DPD estimation process based

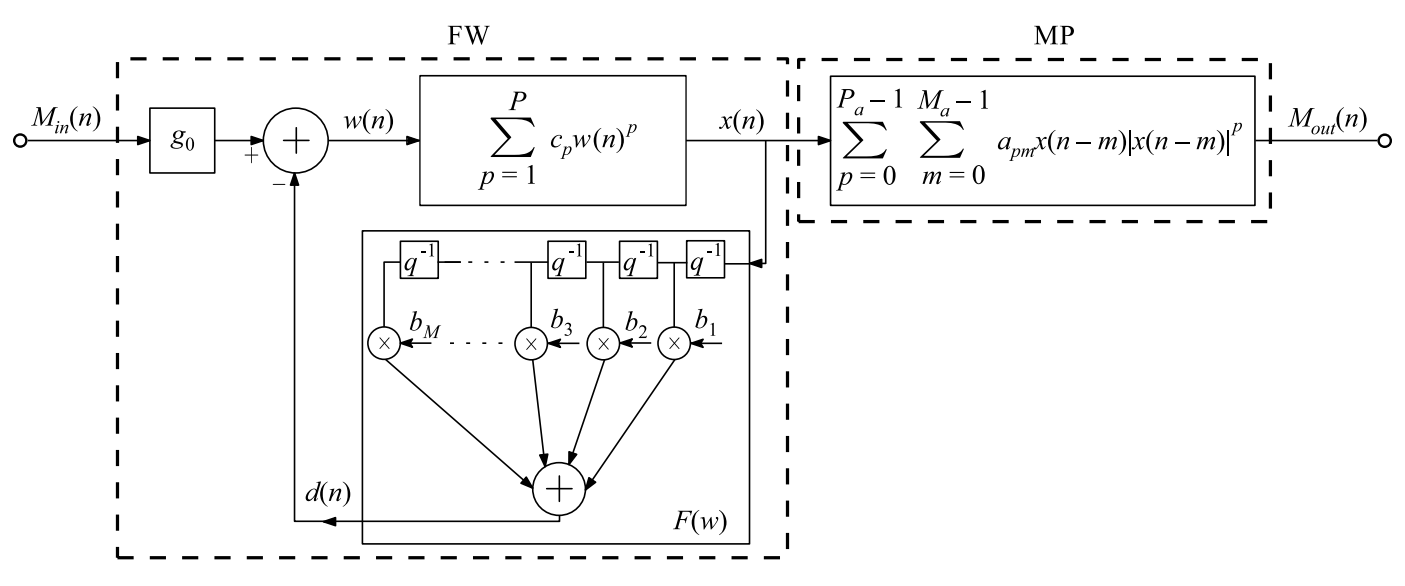

Fig. 1. Feedback Wiener memory polynomial model. 
on the minimization of the quadratic criterion (cost function) according to the output errors - Eq. (5). Note that $G$ is the PA linear gain used for output normalization.

$$
J=\sum_{n=1}^{N} \varepsilon_{i}(n)^{2}+\varepsilon_{Q}(n)^{2},
$$

where $N$ is the length of the signal used (the number of samples).

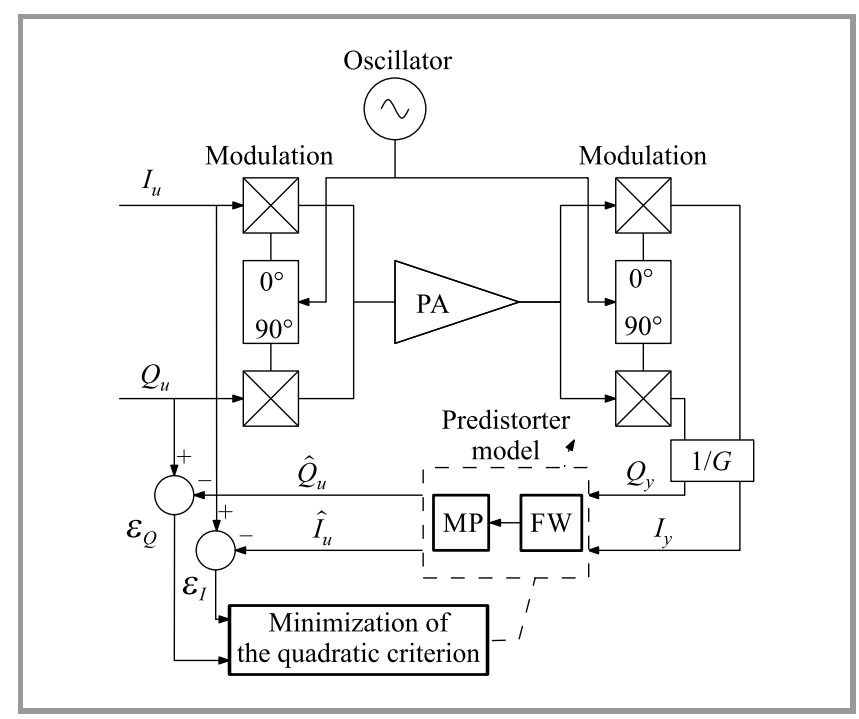

Fig. 2. Offline DPD identification.

As mentioned before, the FWMP model shown in Fig. 1 is composed of two separated blocks, so the identification of such a model class is complex, because of the intermediate unmeasured signals. In other words, the identification of one block requires the simulation of the previous block. In our case, the unmeasured signals are $w(n), x(n)$ and $d(n)$. The key-term separation principle is a good solution for the identification of this model by separating the model into non-linear static and linear dynamic blocks [23]. So, the identification process will be performed in two phases, starting with identification of the FW block followed by the simulation of the intermediate signals. Then, characterization of the MP block will follow.

\subsection{FW Block Identification}

The use of a feedback loop in the FW block renders its onestep identification impossible. We propose, in Fig. 3, an

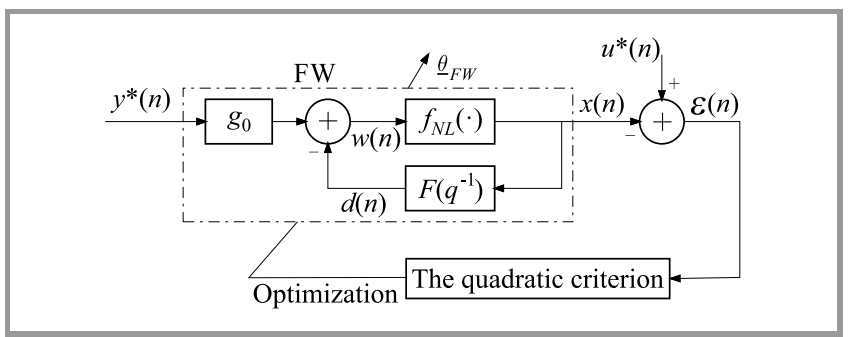

Fig. 3. Identification of the FW block. iterative process to estimate it using the measured PA output and input complex envelope noted by $y^{*}(n)$ and $u^{*}(n)$, respectively. So, in the case of DPD, the measured output data $y^{*}$ will become the input of the FW model $\left(M_{\text {in }}=y^{*}\right)$, and the measured input data $u^{*}$ will become its output. The FW vector of coefficients $\underline{\theta}_{F W}$ is estimated as:

$$
\underline{\theta}_{F W}=\left[\begin{array}{llll}
g_{0} & b_{1} \cdots b_{M} & c_{1} c_{2} \cdots c_{P}
\end{array}\right] .
$$

The FW model for $N$ samples, based on Eqs. (1) and (2), can be rewritten as:

$$
x(n)=\varphi_{F W}^{T}(n, \text { iter }) \cdot \underline{\theta}_{F W},
$$

with:

$$
\begin{aligned}
\varphi_{F W}(n, \text { iter })=\left[\begin{array}{ll}
y^{*}(n) & -x(n-1) \\
& \cdots-x(n-M) \quad w(n) w(n)^{2} \cdots w(n)^{P}
\end{array}\right] .
\end{aligned}
$$

During this iterative process, and in order to avoid the problem of overparametrization [23], we set the first coefficient of the non-linear function $c_{1}$ in Eq. (1) to 1 . Note that during the first iteration of this process, the memory-less function is off. We choose to start the identification process with the estimation of the FIR filter, due to its stability. The iterative identification process of the FW block (Fig. 3) is:

1. Initialization of $w(n), x(n)$ and the FW coefficients Eq. (6), as:

$$
\begin{aligned}
& w(n)=y^{*}(n), \\
& x(n)=u^{*}(n), \\
& \underline{\theta}_{F W}=\left[\begin{array}{lllllll}
1 & 0 & \cdots & 0 & 1 & 0 & \cdots
\end{array}\right]
\end{aligned}
$$

2. Identification of the new feedback filter $F\left(q^{-1}\right)$ coefficient $b_{m}$ and the complex gain $g_{0}$ :

$$
\begin{aligned}
& \underline{\theta}_{F W}(1)=\left[\begin{array}{ll}
g_{0} & b_{1} \cdots b_{M}
\end{array}\right] \text {, } \\
& \varphi_{F W}(n, 1)=\left[y^{*}(n) \quad-x(n-1) \cdots-x(n-M)\right] \text {, }
\end{aligned}
$$

$\underline{\theta}_{F W}(1)$ can be obtained from:

$$
\underline{\theta}_{F W}(1)=\left(\phi^{H} \cdot \phi\right)^{-1} \phi^{H} \cdot x,
$$

where $\phi=\left[\varphi_{F W}^{T}(1,1) \varphi_{F W}^{T}(2,1) \cdots \varphi_{F W}^{T}(N, 1)\right]$.

A QR decomposition function (qrd) is used to avoid matrix inversion problems in $\left(\phi^{H} \cdot \phi\right)$ as:

$$
\operatorname{qrd}\left(\phi^{H} \cdot \phi\right)=Q \cdot R
$$

where $Q$ is an orthogonal unit vectors and $R$ is an upper triangular matrix. Equation (8) becomes:

$$
\underline{\theta}_{F W}(1)=\left(R^{-1} \cdot Q^{H}\right) \phi^{H} \cdot u^{*} .
$$

Based on Eqs. (1)-(2) and using the FW model obtained, we simulate the new intermediate signals noted as $d(n), w(n)$ and $x(n)$; 


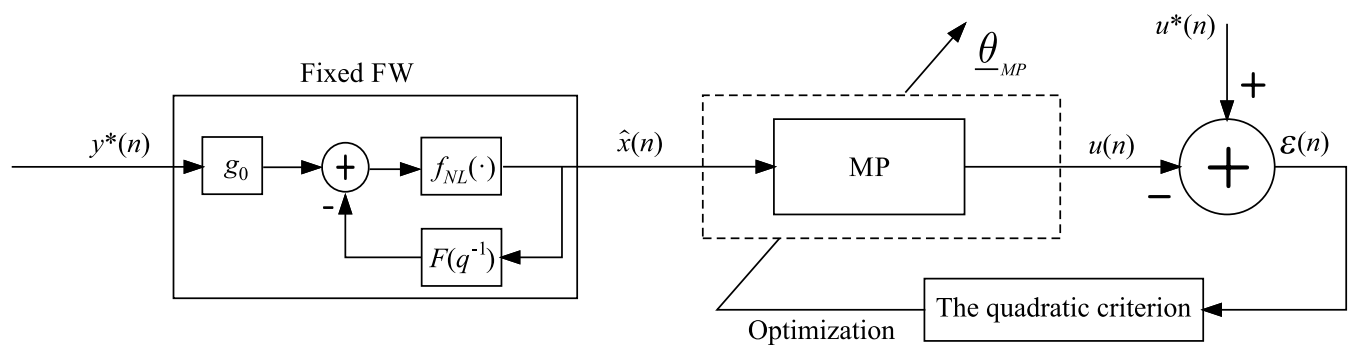

Fig. 4. Identification of the MP block.

3. Estimation of all FW block coefficients $\underline{\theta}_{F W}-$ Eq. (6) using previously simulated intermediate signals, based on Eqs. (7) and (10);

4. Minimization of the quadratic criterion $J=\sum_{n=1}^{N} \varepsilon^{2}(n)$;

5. Repetition of steps 3-4 until a desirable normalized mean square error (NMSE) is reached, or until the adding of another iteration does not reduce the cost function $J$, where:

$$
\mathrm{NMSE}_{d B}=10 \log _{10}\left[\frac{\sum_{n=1}^{N}\left|u^{*}(n)-x(n)\right|^{2}}{\sum_{n=1}^{N}\left|u^{*}(n)\right|^{2}}\right] .
$$

with $x(n)$ being the model output obtained at the last iteration.

\subsection{MP Block Identification}

After the convergence of the FW model, it will be fixed, as shown in Fig. 4, and the simulated output signal $x(n)$ will be used as an input of the MP block.

The MP model coefficients $a_{p m}$ can be obtained using the least squares (LS) algorithm [27] and the system regression derived from relation (3). Thus, for a set of $N$ samples, the optimal coefficients vector $\underline{\theta}_{M P}$ is obtained by solving the following optimization problem:

$$
\min _{\underline{\theta}_{M P}}(J) \quad \text { where } \quad J=\frac{1}{N} \sum_{n=1}^{N}\left|u^{*}(n)-u(n)\right|^{2} .
$$

$u(n)=\varphi_{M P}^{T}\left(n, \underline{\theta}_{M P}\right) \cdot \underline{\theta}_{M P}$ is the model output with:

$$
\begin{gathered}
\varphi_{M P}\left(n, \underline{\theta}_{M P}\right)=\left[x(n) \cdots x(n-m)|x(n-m)|^{p} \cdots\right. \\
\left.x\left(n-M_{a}\right)\left|x\left(n-M_{a}\right)\right|^{P_{a}}\right] \\
\underline{\theta}_{M P}=\left[a_{00} \cdots a_{p m} \cdots a_{\left(P_{a}-1\right)\left(M_{a}-1\right)}\right] .
\end{gathered}
$$

The offline estimation of the $\underline{\theta}_{M P}$ vector is:

$$
\underline{\theta}_{M P}=\left(\phi^{H} \cdot \phi\right)^{-1} \phi^{H} \cdot u^{*},
$$

where $\phi=\left[\varphi_{M P}^{T}\left(1, \underline{\theta}_{M P}\right) \varphi_{M P}^{T}\left(2, \underline{\theta}_{M P}\right) \cdots \varphi_{M P}^{T}\left(N, \underline{\theta}_{M P}\right)\right]$.

\section{Experiments and Results}

\subsection{Experimental Setup and Signal Acquisition}

In this section, we present the experimental validation of the proposed FWMP model and the comparison of its linearization performance with that of a GMP model. The test bench used is shown in Fig. 5. A 2-stages $20 \mathrm{~W}$ class-AB LDMOS RF power amplifier by NXP Semiconductors has been used to validate the proposed model. It has a linear gain of $28 \mathrm{~dB}$ and its $1 \mathrm{~dB}$ compression point is around $41.7 \mathrm{dBm}$, corresponding to an output power backoff (OBO) of $0 \mathrm{~dB}$. A vector signal generator (SMBV100A by Rohde \& Schwarz) is used for up-converting the baseband signal that was generated beforehand using Matlab

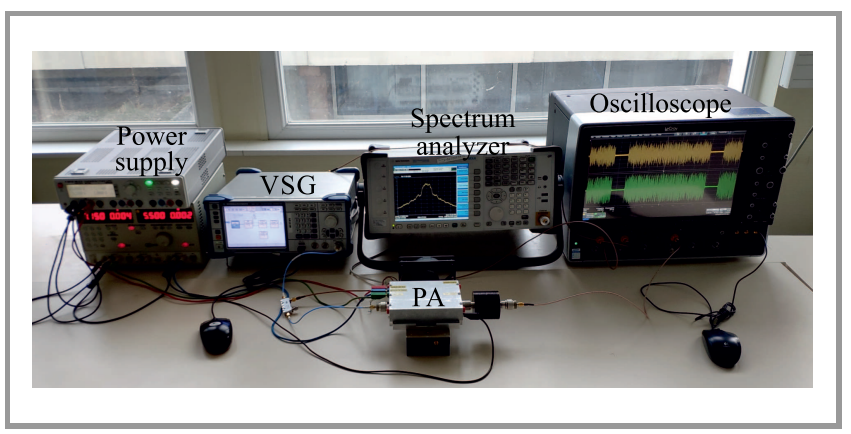

Fig. 5. Experimental setup.

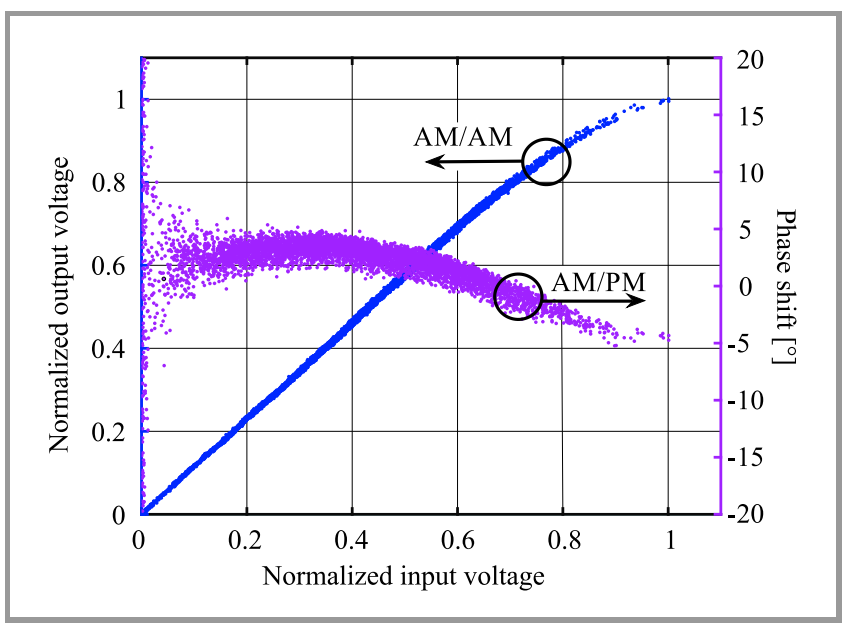

Fig. 6. Instantaneous $\mathrm{AM} / \mathrm{AM}$ and $\mathrm{AM} / \mathrm{PM}$ characteristics for a 64-QAM input signal with $7.5 \mathrm{~dB}$ PAPR. 
and uploaded to the VSG, with a carrier frequency of 3.7 GHz. The sequence used is a filtered $10 \mathrm{MHz} 64-$ QAM modulated signal with a peak-to-average power ratio (PAPR) of approximately $7.5 \mathrm{~dB}$.

At the PA output, the RF signal was acquired at a sampling frequency of $40 \mathrm{GHz}$ and then numerically down-converted and demodulated using a 4-channels oscilloscope (LeCroy WaveMaster $816 \mathrm{Zi}-\mathrm{A})$. The input and output baseband signals are synchronized in the time domain using Matlab.

Non-linearities and memory effects of the used PA may be observed from the dynamical AM/AM and AM/PM functions in Fig. 6. We can see that the gain is compressed by PA when the input level increases.

\subsection{Experimental Results}

In our study, the merit value refers to the NMSE criterion given by Eq. (11) which translates the modeling accuracy. So, to determine the DPD structure using the FWMP model in terms of the trade-off between performances and complexity, an exhaustive search is performed in Fig. 7.

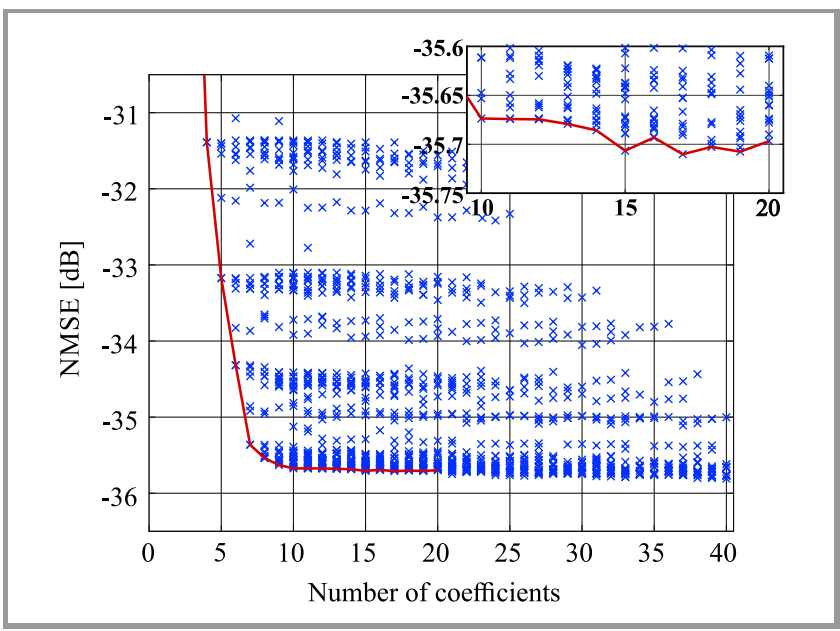

Fig. 7. Determination of the most relevant FWMP model structure.

We mapped NMSE in the time-domain versus the number of coefficients by testing all possible structures. A single set of input and output signals measured is used in this mapping and is the same during the process of identifying all structures. Each point in the map corresponds to an NMSE value using a set of FWMP parameters $P, M, P_{a}$ and $M_{a}$ - see Eqs. (1)-(3). Here, 1715 combinations were tested with $P=1: 7, M=0: 4, P_{a}=1: 7$, and $M_{a}=1: 7$. During the optimization phase, we determined that it takes less than 10 iterations for the model to converge to the lowest NMSE, so for each structure, we iterated the model 10 times to obtain the final coefficients.

As shown in this NMSE map, an increase in the number of coefficients allows to improve estimation performance. It may also be noticed that for the same number of coefficients, several values of NMSE may be identified. For example, for structures with 10 coefficients, the lowest
NMSE of approx. $-35.6 \mathrm{~dB}$, is obtained with the model orders $\left(P=7, M=2, P_{a}=1, M_{a}=1\right)$, while the worst result of approx. $-22 \mathrm{~dB}$ is obtained for $\left(P=1, M=2, P_{a}=1\right.$, $\left.M_{a}=7\right)$. These results show the importance of an offline DPD evaluation determining the best FWMP structure, i.e. using a minimum number of coefficients for a given NMSE requirement.

Figure 8 shows the parameter orders of the FWMP models, with different numbers of coefficients (up to 20 coefficients), which ensure the best performance in terms of NMSE.

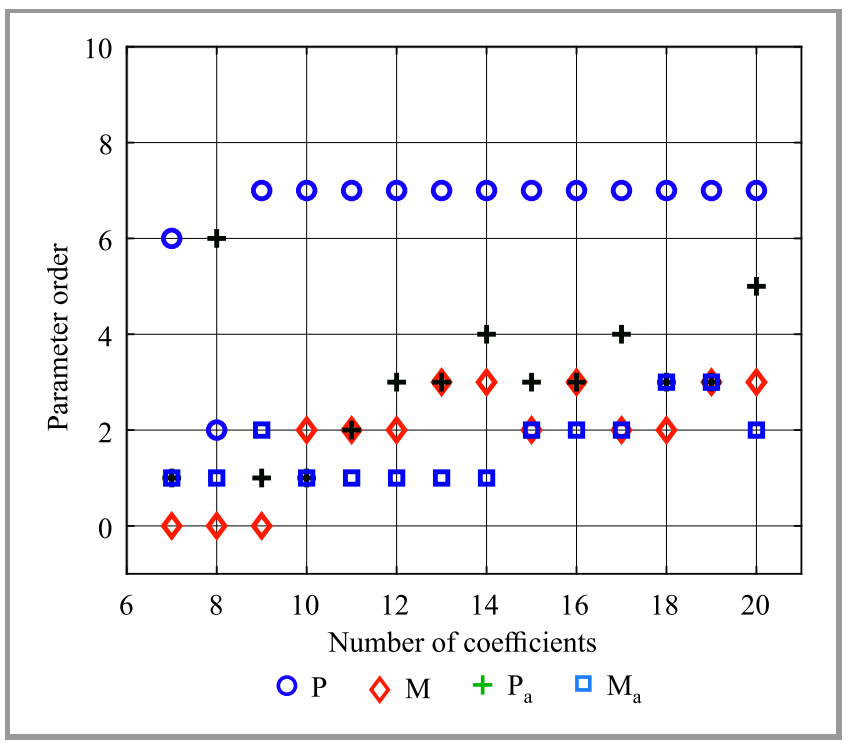

Fig. 8. Composition of the best structures obtained with different numbers of coefficients.

We can see from Fig. 8 that the FW block is the one that contributes the most to the description of the DPD function. An FW non-linearity order $P$ of 7 is sufficient to describe the non-linear behavior of the PA used. Also, for structures with up to 10 coefficients, we realize that it is more relevant to use only the FW block, while starting from 11 coefficients, the deployment of the MP block provides better performance.

Table 1

Comparison of complexity (number of FLOP) and performance of different model structures

\begin{tabular}{|c|c|c|c|c|c|}
\hline \multirow{4}{*}{} & \multirow{2}{*}{$\mathrm{P}$} & \multirow{2}{*}{$\mathrm{M}$} & \multirow{2}{*}{ NMSE [dB] } & \multicolumn{2}{|c|}{ No. of FLOPs } \\
\cline { 5 - 6 } & & & & Add. & Multipl. \\
\hline \multirow{4}{*}{ NL } & 7 & 0 & -35.53 & 6 & 28 \\
\cline { 2 - 6 } & 8 & 0 & -35.57 & 7 & 36 \\
\cline { 2 - 6 } & 9 & 0 & -35.64 & 8 & 45 \\
\cline { 2 - 6 } & 10 & 0 & -35.64 & 9 & 55 \\
\cline { 2 - 6 } & 11 & 0 & Unstable & 10 & 66 \\
\hline FW & 7 & 2 & -35.67 & 8 & 31 \\
\hline
\end{tabular}

To show the importance of using the feedback loop (FIR filter), in Table 1 a comparison between complexity (num- 
ber of FLOPs) and performance of the individual cases is presented, in which:

- only the memoryless polynomial is used,

- only the FW is used.

A floating point operation (FLOPs) describes the arithmetic operation on floating point numbers.

We can see from Table 1 that the best NMSE with only the NL function, close to the one obtained with the complete FW block, is obtained with a non-linearity order of 10, but at the cost of a significant increase in the number of FLOPs. Moreover, the NL function becomes unstable starting from the non-linearity order of 11 . It also needs to be noted that the introduction of the feedback filter helps improve the NMSE, with only a slight increase in the number of FLOPs.

By tracking the evolution of the lowest NMSE in each column (red line in Fig. 7), we can note that there is no significant enhancement of the NMSE after 10 coefficients. Zooming on the area between 10 and 20 coefficients enables a precise measuring, which helped us choose a structure with 15 coefficients as a point of reference for our study. This structure, obtained with $(P=7, M=2$, $P_{a}=3, M_{a}=2$ ), offers a good trade-off between modeling accuracy and model complexity.

\subsection{Comparison of the FWMP Structure with the MP and GMP Models}

To show the importance of the FW block in the proposed cascaded model, we performed a set of experiments - both with and without the FW block. Thus, partial mapping was performed using the MP model only (Eq. (3)) with 10 to 20 coefficients (see red triangles in Fig. 9).

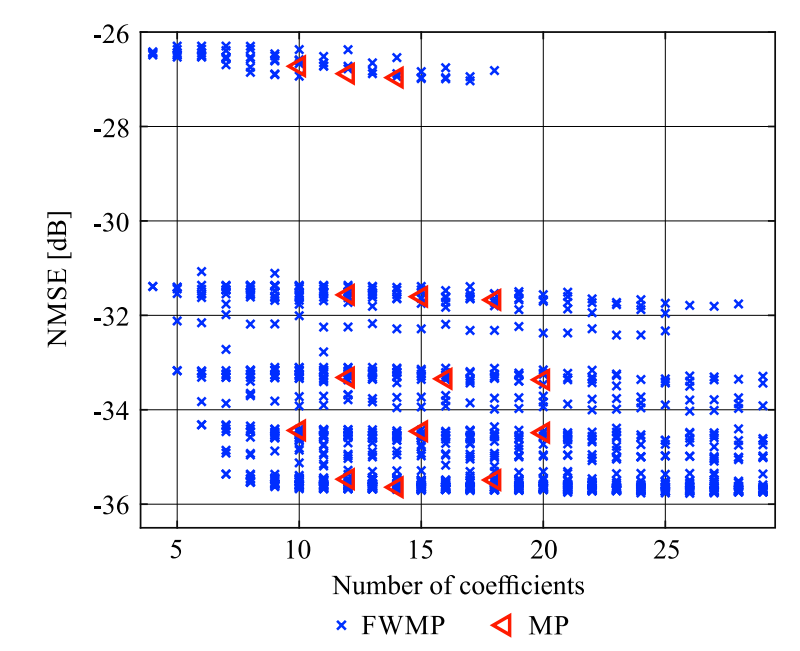

Fig. 9. Comparison of FWMP and MP models.

As one may notice in Fig. 9, introduction of the FW block in the general case allows to reach a lower NMSE. So, whatever the number of coefficients is, the FWMP model allows to improve NMSE. For example, in the reference case of a model with 15 coefficients, the FW introduces additional dynamics that improve the NMSE by approx. $1 \mathrm{~dB}$.

Below, we compare FWMP with a GMP model [21].

As a reminder, the GMP model is given by the following relation:

$$
\begin{aligned}
& M_{\text {out }}(n)=\sum_{p=0}^{P_{a}-1} \sum_{m=0}^{M_{a}-1} a_{p m} \cdot M_{\text {in }}(n-m) \cdot\left|M_{\text {in }}(n-m)\right|^{p} \\
& +\sum_{p=1}^{P_{b}} \sum_{m=0}^{M_{b}-1} \sum_{l=1}^{L_{b}} b_{p m l} \cdot M_{\text {in }}(n-m) \cdot\left|M_{\text {in }}(n-m-l)\right|^{p} \\
& +\sum_{p=1}^{P_{c}} \sum_{m=0}^{M_{c}-1} \sum_{l=1}^{L_{c}} c_{p m l} \cdot M_{\text {in }}(n-m) \cdot\left|M_{\text {in }}(n-m+l)\right|^{p}
\end{aligned}
$$

and its number of coefficients $N_{G M P}$ may be obtained by:

$$
N_{G M P}=P_{a} \cdot M_{a}+P_{b} \cdot M_{b} \cdot L_{b}+P_{c} \cdot M_{c} \cdot L_{c} .
$$

In order to compare FWMP with the GMP model, we deployed them under the same conditions, and plotted the obtained NMSE with structures comprising from 1 to 50 coefficients. The purple dots show the results for the GMP model (Fig. 10). In this case, 345945 combinations were tested with $P_{a}=1: 7, M_{a}=1: 4, P_{b}=1: 7, M_{b}=1: 7, L_{b}=$ $1: 5, P_{c}=1: 7, M_{c}=1: 7, L_{c}=1: 5$.

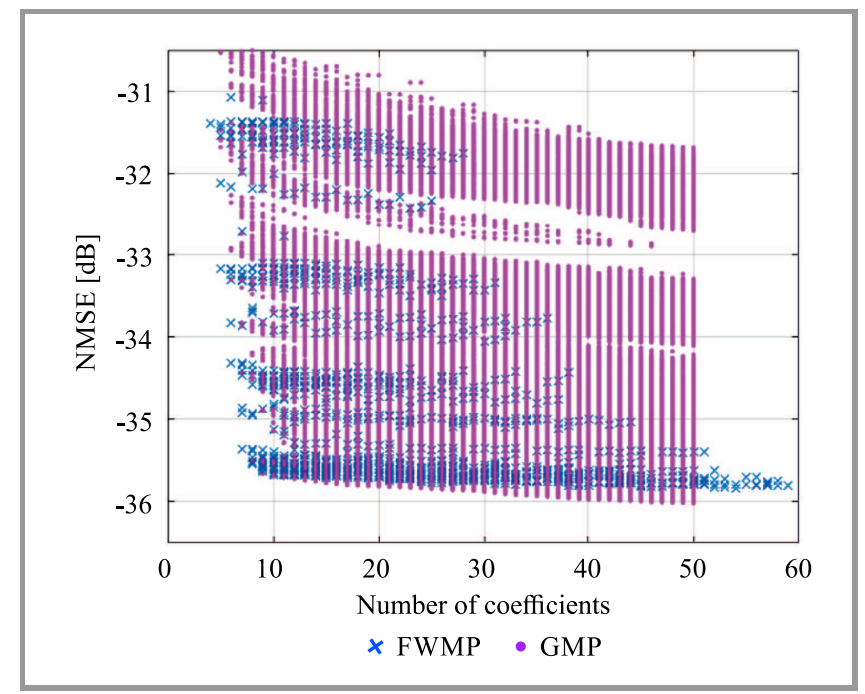

Fig. 10. Comparison of FWMP and GMP models.

The first remark concerns the greater number of potential structures to be tested, observed in the case of the GMP model. In fact, and as expressed in Eq. (15), there are 8 sizing parameters applicable to the GMP model: nonlinearity orders $\left(P_{a}, P_{b}\right.$ and $\left.P_{c}\right)$, memory depths $\left(M_{a}, M_{b}\right.$, and $M_{c}$ ), and lagging and leading delay tap lengths $\left(L_{b}\right.$ and $\left.L_{c}\right)$. That is the major drawback of the GMP model, where the number of combinations increases rapidly along with the model orders. In the case of FWMP from Eqs. (1)-(3), we reduce the number of sizing parameters to 4: non-linearity orders $\left(P\right.$ and $P_{a}$ ), filter order $M$ and memory depth $M_{a}$. 


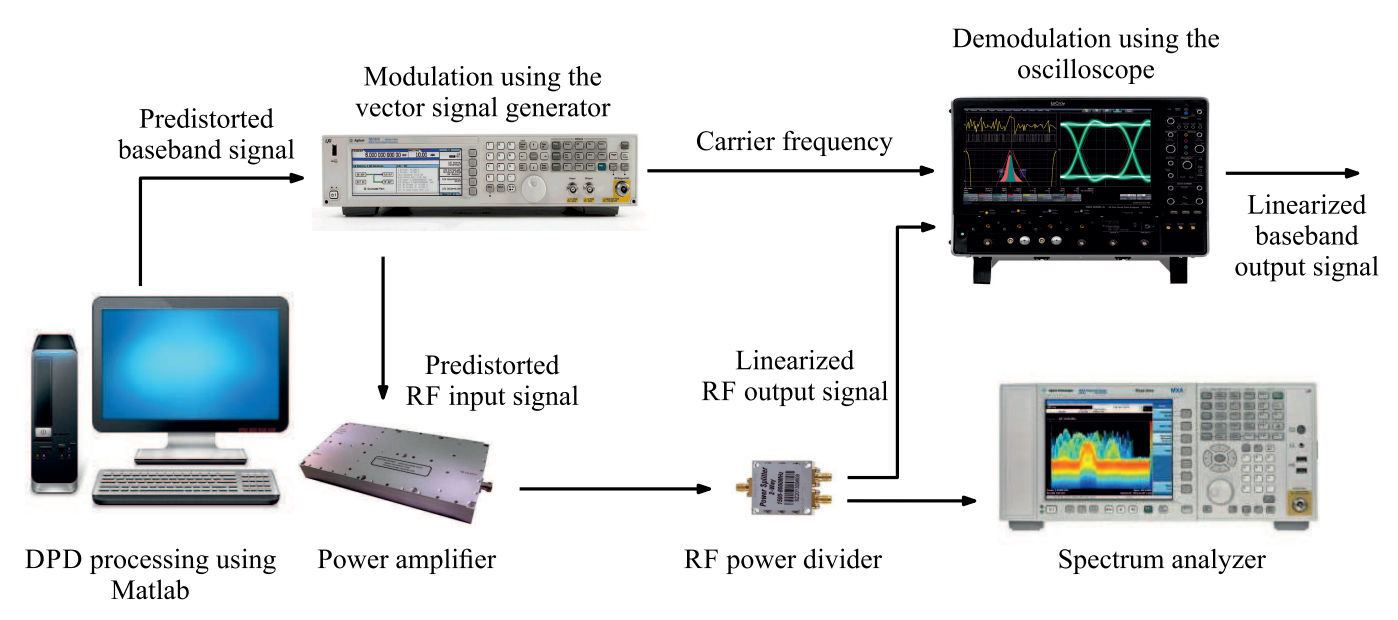

Fig. 11. Linearization system scheme.

The second remark concerns the performance of both models. As can be concluded from Fig. 10, for structures with up to 27 coefficients, both models offer almost the same performance, with the FWMP model having the advantage of low complexity. Beyond that, the GMP model shows a slight improvement, for example of $0.23 \mathrm{~dB}$ in the case of a structure with 50 coefficients.

Based on these comparisons, the best GMP and FWMP structures with 15 coefficients, allowing the lowest NMSE, have been extracted and used as a DPD function to linearize the PA behavior. The number of arithmetic operations of each model is presented in Table 2.

Table 2

Comparison in terms of complexity (number of FLOPs) of the two used models

\begin{tabular}{|c|c|c|c|c|c|}
\hline \multirow{2}{*}{ Model } & \multirow{2}{*}{ Model structure } & \multicolumn{2}{|c|}{$\begin{array}{c}\text { FLOPs } \\
\text { per block }\end{array}$} & \multicolumn{2}{|c|}{ Total } \\
\cline { 3 - 5 } & & Add. & Multipl. & Add. & Multipl. \\
\hline \multirow{2}{*}{ FWMP } & $P=7, M=2$ & 8 & 31 & \multirow{2}{*}{13} & \multirow{2}{*}{43} \\
\cline { 2 - 4 } & $P_{a}=3, M_{a}=2$ & 5 & 12 & & \\
\hline \multirow{3}{*}{ GMP } & $P_{a}=7, M_{a}=1$ & 6 & 28 & \multirow{3}{*}{$12+2$} & \multirow{2}{*}{48} \\
\cline { 2 - 5 } & $P_{b}=2, M_{b}=2, L_{b}=1$ & 3 & 10 & & \\
\cline { 2 - 5 } & $P_{c}=2, M_{c}=1, L_{c}=2$ & 3 & 10 & & \\
\hline
\end{tabular}

As shown in Table 2, and in comparison with the GMP model, the proposed FWMP model is characterized by a lower number of arithmetic operations.

Figure 11 illustrates the experimental process in which the input signal is predistorted using Matlab software and then uploaded to VSG, which provides the RF predistorted signal to be injected to the PA. Both time and frequency experiments are performed for FWMP and GMP models under the same conditions. Review of these results allows to determine the contribution of the proposed model.

In the time-domain, the linearized AM/AM and AM/PM characteristics of the LDMOS PA used, obtained using the two models, namely FWMP and GMP, are shown in Figs. 12 and 13, respectively. We can see that the FWMP

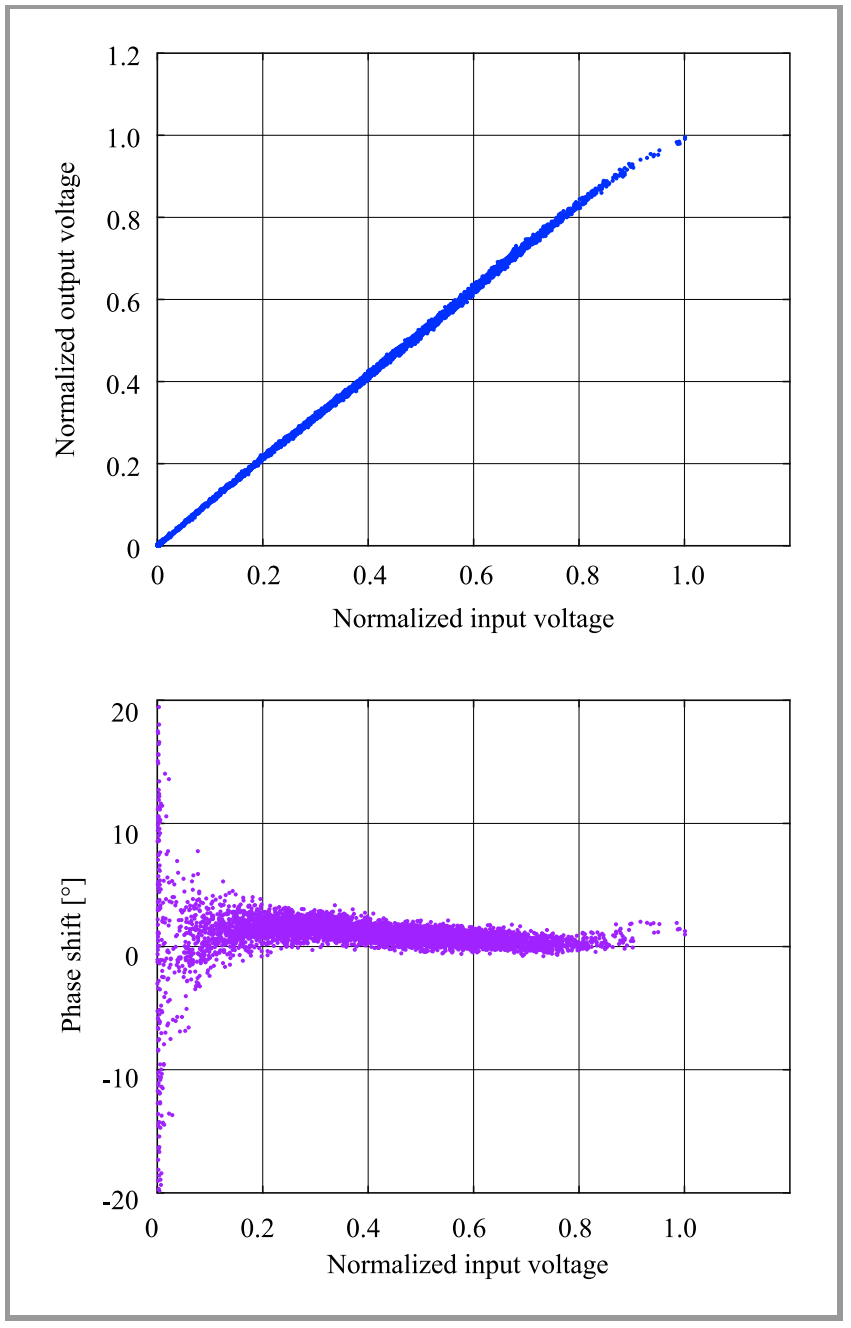

Fig. 12. Linearized AM/AM and AM/PM characteristics using FWMP model.

model offer good linearization performance, similar to that of the GMP structure. No significant differences between the studied models are noticed and the obtained results show the robustness and the effectiveness of both solutions. 


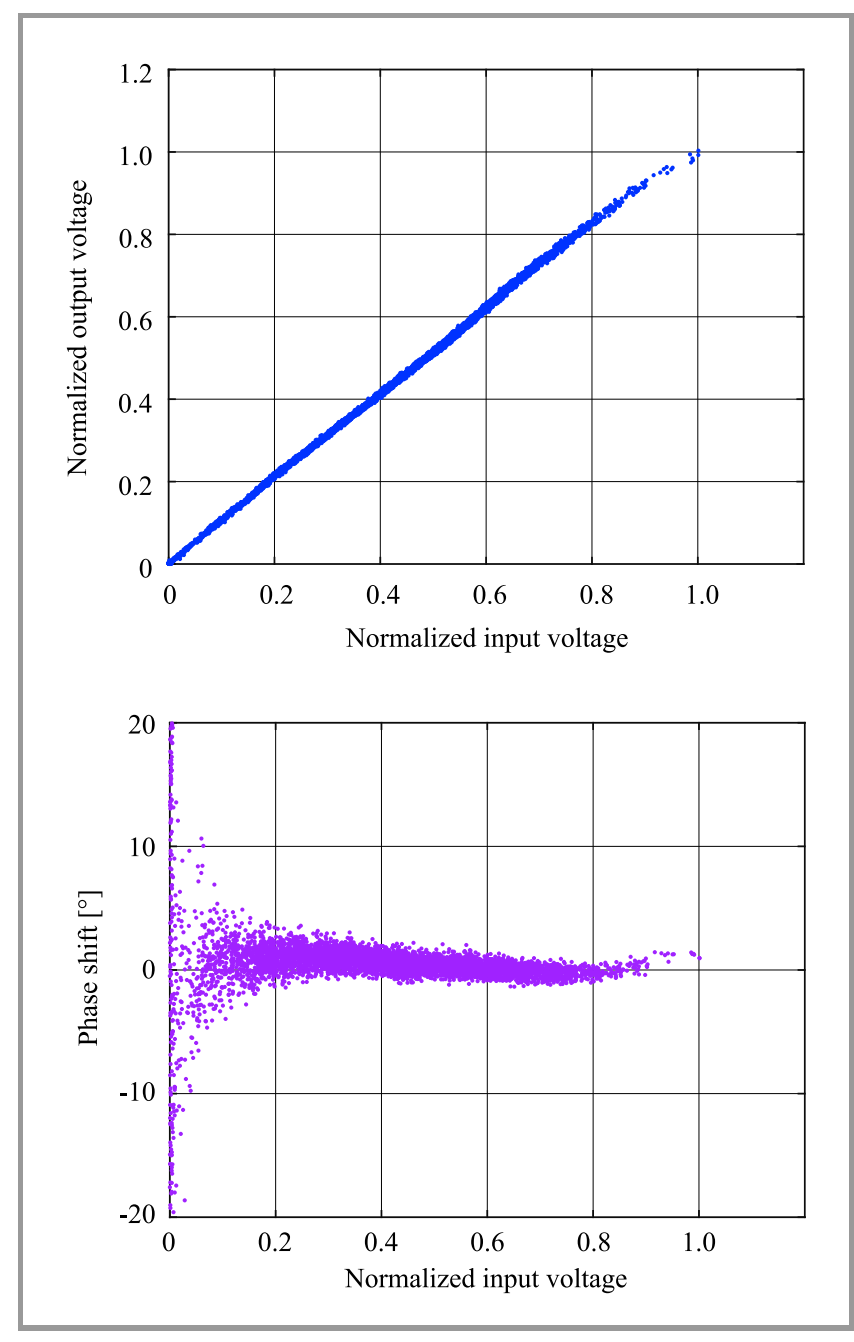

Fig. 13. Linearized $\mathrm{AM} / \mathrm{AM}$ and $\mathrm{AM} / \mathrm{PM}$ characteristics using GMP model.

\subsection{Results in Frequency Domain}

It is known that the PA non-linearities create spectral regrowth distortions and adjacent channel noise [28]. To determine the performance of FWMP in the frequency domain, in Fig. 14, we present the measured PA output spectra, both with and without DPD, using the two models. According to the obtained spectra, we may see in Fig. 14 that the linearization performance of both models is similar and that the suppression of sideband noises caused by PA non-linearities and memory effects is effective.

Table 3 presents a comparison between the adjacent channel power ratio (ACPR) obtained using the two models and different adjacent channel bandwidths $\Delta W_{L / U}$ (5 and $10 \mathrm{MHz}$ ). As a reminder, ACPR at the PA output is based on the discrete Fourier transform $Y(\omega)$ of $y(n)$ and is used to evaluate out-of-band distortion, provided that $L$ and $U$ are the lower and the upper adjacent channel frequencies, respectively. $M$ is the main channel frequency.

$$
A C P R_{d B c}=\frac{\int_{\Delta W_{M}} Y(\omega) \mathrm{d} \omega}{\int_{\Delta W_{L / U}} Y(\omega) \mathrm{d} \omega},
$$

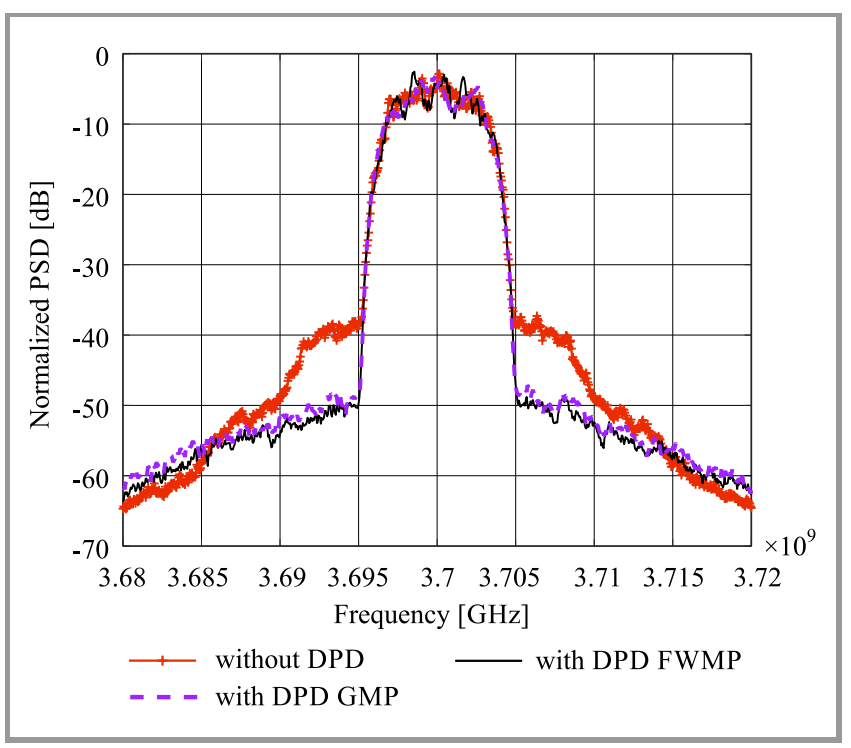

Fig. 14. Output spectra measured using FWMP and GMP linearizer.

Table 3

Comparison of ACPR achieved

\begin{tabular}{|c|c|c|c|c|}
\hline$\Delta W[\mathrm{MHz}]$ & ACPR $[\mathrm{dB}]$ & Original & DPD GMP & DPD FWMP \\
\hline 5 & Lower & -32.82 & -42.37 & -43.77 \\
\hline 5 & Upper & -32.59 & -41.66 & -42.84 \\
\hline 10 & Lower & -35.52 & -43.84 & -45.16 \\
\hline 10 & Upper & -35.31 & -43.45 & -44.56 \\
\hline
\end{tabular}

Results shown in Table 3 confirm those obtained in Fig. 14, where the performance of GMP and FWMP was similar, and allow for a $10 \mathrm{~dB}$ ACPR improvement compared with the original signal. These results confirm the contribution of the FWMP structure in sizing the optimal linearizer.

\section{Conclusion}

Power amplifier non-linearities and memory effects have been discussed in this paper, and a new cascaded structure has been proposed as a low complexity linearizer. This model, based on the feedback Wiener system, ensures the correction and an overall improvement of the spectral distortions over a wide range of frequency bands.

The measurement system and the identification method were presented as well. Experimental results obtained for a commercial class-AB LDMOS PA by NXP Semiconductors demonstrated that the performance of the FWMP structure is better than that of the MP model and similar to that of the GMP model. Spectral analysis also shows an improvement in the out-of-band emission by up to $10 \mathrm{~dB}$ of ACPR, which may increase the power efficiency of the transmitter. The proposed model contributes to reducing the number of parameters, which is a considerable gain in term of the number of combinations to be tested during the model identification process.

We can finally conclude that the FWMP model may outperform other DPD structures in reducing spectral regrowth 
or ACPR, without increasing model complexity. However, since the proposed model is a 2 -stage cascaded DPD, identification of its coefficients is more complicated. To deal with this drawback, a new way to identify its complex coefficients, based on iterative estimation, is proposed. Future work will focus on using the DPD FWMP model in on-line identification for a reconfigurable PA, where different learning architectures may be used for stage-by-stage identification.

\section{Acknowledgement}

Research activities described in this publication are part of the APOGEES project supported by BPI France, region Occitanie and region Nouvelle Aquitaine. The APOGEES project has been labeled by pole Aerospace Valley, pole I\&R and pole Alpha-RLH in the framework of the French FUI22 research program.

\section{References}

[1] P. Reynaert and M. Steyaert, "Mobile communication systems and power amplification", in RF Power Amplifiers for Mobile Communications. Springer Science \& Business Media, 2006, pp. 9-64 (ISBN: 9781402051166).

[2] A. A. M. Saleh, "Frequency-independent and frequency-dependent nonlinear models of TWT amplifiers", IEEE Trans. on Commun., vol. 29, no. 11, pp. 1715-1720, 1981 (DOI: 10.1109/TCOM.1981.1094911).

[3] J. Vuolevi, T. Rahkonen, and J. Manninen, "Measurement technique for characterizing memory effects in RF power amplifiers", IEEE Trans. on Microw. Theory and Techniq., vol. 49, no. 8, pp. 1383-1389, 2001 (DOI: 10.1109/22.939917).

[4] H. Ku, M. D. Mckinley, and J. Kenney, "Quantifying memory effects in RF power amplifiers", IEEE Trans. on Microw. Theory and Techniq., vol. 50, no. 12, pp. 2843-2849, 2002 (DOI: 10.1109/TMTT.2002.805196).

[5] E. Ngoya, C. Quindroit, and J. M. Nebus, "On the continuous-time model for nonlinear memory modeling of RF power amplifiers", IEEE Trans. on Microw. Theory and Techniq., vol. 57, no. 12, pp. 3278-3292, 2009 (DOI: 10.1109/TMTT.2009.2033297).

[6] C. S. Aitchison, M. Mbabele, M. R. Moazzam, D. Budimir, and F. Ali, "Improvement of third-order intermodulation product of RF and microwave amplifiers by injection", IEEE Trans. on Microw. Theory and Techniq., vol. 49, pp. 1148-1154, 2001 (DOI: 10.1109/22.925508).

[7] Y. Aimer, B. S. Bouazza, S. Bachir, and C. Duvanaud, "Interleaving technique implementation to reduce PAPR of OFDM signal in presence of non-linear amplification with memory effects", $J$. of Telecommun. and Inform. Technol., no. 3, 2018 (DOI: 10.26636/jtit.2018.123517).

[8] M. Vaskovic, "Compensation of nonlinear distortion in RF amplifiers for mobile communications", $\mathrm{Ph} . \mathrm{D}$. thesis, University of Westminster, London, England, 2014 [Online]. Available: https://westminsterresearch.westminster.ac.uk/item/8yvxv/ compensation-of-nonlinear-distortion-in-rf-amplifiers-for-mobilecommunications

[9] S. Bachir, C. E. Nicusor, and C. Duvanaud, "Linearization of RF power amplifiers using adaptive Kalman filtering algorithm", J. of Circ., Syst., and Computers, vol. 20, no. 6, pp. 1001-1018, 2011 (DOI: 10.1142/S0218126611007724).

[10] C. Nader, P. N. Landin, W. Van Moer, N. Bjorsell, and P. Handel, "Performance evaluation of peak-to-average power ratio reduction and digital pre-distortion for OFDM based systems", IEEE Trans. on Microw. Theory and Techniq., vol. 59, no. 12, pp. 3504-3511, 2011 (DOI: 10.1109/TMTT.2011.2170583).
[11] I. Teikari, "Digital predistortion linearization methods for RF power amplifiers", Ph.D. thesis, Aalto University of Technology, Espoo, Finland, 2008 [Online]. Available: http://lib.tkk.fi/Diss/2008/ isbn9789512295463/isbn9789512295463.pdf

[12] M. A. Hussein, Y. Wang, G. Peyresoubes, B. Feuvrie, and S. Toutain, "LUT/parametric digital predistortion approach for the linearization of power amplifiers characteristics", in Proc. 38th Eur. Microwave Conf. EuMC 2008, Amsterdam, Netherlands, 2008, pp. 571-574 (DOI: 10.1109/EUMC.2008.4751516).

[13] P. Banelli and G. Baruffa, "Mixed BB-IF predistortion of OFDM signals in non-linear channels", IEEE Trans. on Broadcast., vol. 47, pp. 137-146, 2001 (DOI: 10.1109/11.948266).

[14] X. Feng, "Efficient baseband digital predistortion techniques for linearizing power amplifier by taking into account nonlinear memory effect", Ph.D. thesis, University of Nantes, Nantes, France, 2015 [Online]. Available: https://hal.archives-ouvertes.fr/tel-01206266

[15] L. Ding, "Digital predistortion of power amplifiers for wireless applications", Ph.D. Thesis, Georgia Institute of Technology, Georgia, USA, 2004 [Online]. Available: https://smartech.gatech.edu/ bitstream/handle/1853/5184/ding_lei_200405_phd.pdf

[16] V. Volterra, Theory of Functionals and of Integral and IntegroDifferential Equations". London: Blackie \& Son Ltd, 1930.

[17] C. Yu, L. Guan, E. Zhu, and A. Zhu, "Band-limited Volterra seriesbased digital predistortion for wideband RF power amplifiers", IEEE Trans. on Microw. Theory and Techniq., vol. 60, pp. 4198-4208, 2012 (DOI: 10.1109/TMTT.2012.2222658).

[18] H. E. Hamoud, T. Reveyrand, S. Mons, and E. Ngoya, "A comparative overview of digital predistortion behavioral modeling for multi-standards applications", in Proc. Int. Worksh. on Integr. Nonlin. Microw. and Millim.-wave Circ. INMMIC 2018, Brive La Gaillarde, France, 2018 (DOI: 10.1109/INMMIC.2018.8430010).

[19] J. Kim and K. Konstantinou, "Digital predistortion of wideband signals based on power amplifier model with memory", Electron. Lett., vol. 37, no. 23, pp. 1417-1418, 2001 (DOI: 10.1049/el:20010940).

[20] S. Amin, P. Landin, P. Händel, and D. Rönnow, "2D extended envelope memory polynomial model for concurrent dual-band RF transmitters", Int. J. of Microw. and Wirel. Technol., vol. 9, no. 8, pp. 1619-1627, 2017 (DOI: 10.1017/S1759078717000277).

[21] D. R. Morgan, Z. Ma, J. Kim, M. G. Zierdt, and J. Pastalan, "A generalized memory polynomial model for digital predistortion of RF power amplifiers", IEEE Trans. on Sig. Process., vol. 54, no. 10, pp. 3852-3860, 2006 (DOI: 10.1109/TSP.2006.879264).

[22] P. L. Gilabert, A. Cesari, G. Montoro, E. Bertran, and J. Dilhac, "Multi-lookup table FPGA implementation of an adaptive digital predistorter for linearizing RF power amplifiers with memory effects", IEEE Trans. on Microw. Theory and Techniq., vol. 56, no. 2, pp. 372-384, 2008 (DOI: 10.1109/TMTT.2007.913369).

[23] F. Guo, "A new identification method for Wiener and Hammerstein systems", Ph.D. thesis, University of Karlsruhe, Germany, 2003 [Online]. Available: https://digbib.ubka.uni-karlsruhe.de/volltexte/ fzk/6955/6955.pdf

[24] S. Afsardoost, T. Eriksson, and C. Fager, "Digital predistortion using a vector-switched model", IEEE Trans. on Microw. Theory and Techniq., vol. 60, no. 4, pp. 1166-1174, 2012 (DOI: 10.1109/TMTT.2012.2184295).

[25] A. Zhu, "Decomposed vector rotation-based behavioral modeling for digital predistortion of RF power amplifiers", IEEE Trans. on Microw. Theory and Techniq., vol. 63, no. 2, pp. 737-744, 2015 (DOI: 10.1109/TMTT.2014.2387853).

[26] T. H. C. Bouazza, S. Bachir, and C. Duvanaud, "Behavioral blocks model for complexity-reduced modeling of RF power amplifiers", in Proc. of the IEEE Int. Symp. on Circ. and Syst. ISCAS 2019, Sapporo, Japan, 2019 (DOI: 10.1109/ISCAS.2019.8702517).

[27] B. Friedlander and M. Morf, "Least squares algorithms for adaptive linear-phase filtering", IEEE Trans. on Acoust., Speech, and Sig. Process., vol. 30, no. 3, pp. 381-390, 1982 (DOI: 10.1109/TASSP.1982.1163903).

[28] A. Cheaito, "Analytical analysis of in-band and out-of-band distortions for multicarrier signals: Impact of non-linear amplification, memory effects and predistortion", Ph.D. thesis, INSA Rennes, University of Bretagne Loire, Rennes, France, 2017 [Online]. Available: https://www.theses.fr/2017ISAR0001.pdf 


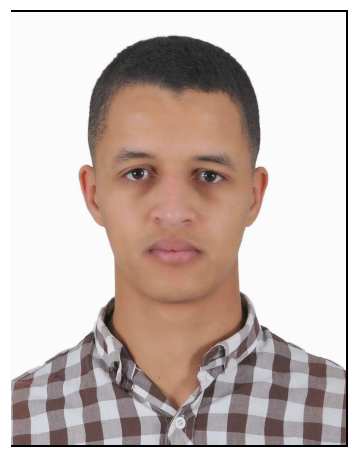

Tayeb H. C. Bouazza received his B.Sc. and M.Sc. degrees in Electronics and Telecommunications from the University of Saïda, Algeria in 2011 and 2013, respectively. Since 2017, he has been a Ph.D. student focusing on electronics, microelectronics, nanoelectronics and microwaves at the XLIM laboratory, Department of Smart Networks and Systems, University of Poitiers, France. His current research interests are in modeling non-linear systems, signal processing and wireless communication.

(iD) https://orcid.org/0000-0003-3873-0087

E-mail: tayeb.habib.chawki.bouazza@univ-poitiers.fr

XLIM Laboratory UMR-CNRS 7252

Institute of Technology of Angoulême

University of Poitiers

4 avenue de Varsovie

16000 Angoulême, France

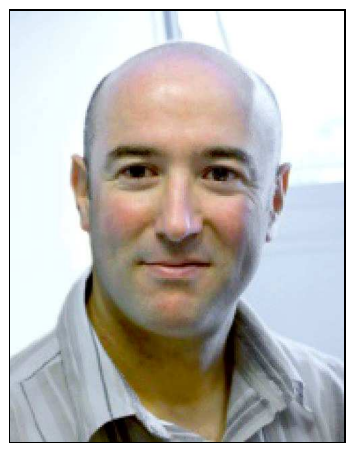

Smail Bachir received B.Sc. and M.Sc. degrees in Signal Theory from the Polytechnic School of Algeria in 1997. He joined the scientific department of Leroy Somer Society and the University of Poitiers in France, where he received his Ph.D. degree in Automatic and Electrical Engineering in 2002 and the habilitation de- gree (HDR) in 2015. He is presently an Associate Professor at the University of Poitiers and a researcher at XLIM laboratory with the Department of Smart Networks and Systems. His research interests include signal processing, nonlinear systems parameter identification, electronic devices and wireless circuits.

E-mail: smail.bachir@univ-poitiers.fr

XLIM Laboratory UMR-CNRS 7252

Institute of Technology of Angoulême

University of Poitiers

4 avenue de Varsovie

16000 Angoulême, France

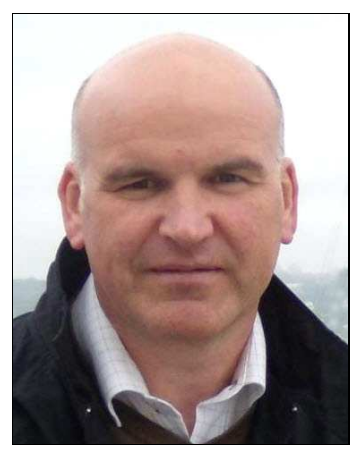

Claude Duvanaud received his Ph.D. in Electronics and Communication Engineering from the University of Limoges, France, in 1993 and the habilitation degree (HDR) from the University of Poitiers in 2003. Currently, he is an Associate Professor at the University of Poitiers and XLIM laboratory, France. His research interests include modeling, simulation and design of non-linear power amplifiers and communication systems.

E-mail: claude.duvanaud@univ-poitiers.fr

XLIM Laboratory UMR-CNRS 7252

Institute of Technology of Angoulême

University of Poitiers

4 avenue de Varsovie

16000 Angoulême, France 